\title{
REMARKS ON DIFFERENTIAL INEQUALITIES IN BANACH SPACES ${ }^{1}$
}

\author{
ROBERT H. MARTIN, JR.
}

ABSTRACT. Under certain conditions a comparison is made between solutions of a pair of initial value problems in a Banach space. This comparison includes and unifies several recent results on differential inequalities in Banach spaces.

Let $E$ be a real or complex Banach space with the norm on $E$ denoted 1. 1. Suppose that $T>0$ and that $A$ and $B$ are continuous functions from $[0, T] \times E$ into $E$. In this note we consider differential inequalities and invariance properties associated with the initial value problems

$$
u^{\prime}(t)=A(t, u(t)), \quad u(\lambda)=z, \quad \lambda \leq t \leq T,
$$

and

$$
v^{\prime}(t)=B(t, v(t)), \quad v(\lambda)=w, \quad \lambda \leq t \leq T,
$$

where $0 \leq \lambda<T$ and $z$ and $w$ are in $E$. The results presented here have considerable overlap with recent works in this area by Redheffer, Volkmann and Walter. See, e.g., [5]-[7] and the results of Bittner [1].

Suppose that $z \in E, \mathcal{E}>0$ and $\lambda, \mu \in[0, T]$ with $\lambda<\mu$. A continuous function $\alpha=a(\cdot ; \lambda, \mu, z, \mathcal{E})$ from $[\lambda, \mu]$ into $E$ is said to be an $\mathscr{E}$-approximate solution to (1) on $[\lambda, \mu]$ if $\alpha(\lambda)=z$ and $\alpha_{+}^{\prime}(t)$ exists with $\mid \alpha_{+}^{\prime}(t)-$ $A \alpha(t) \mid \leq \mathcal{E}$ for all $t \in[\lambda, \mu)$. We also suppose that $\alpha_{+}^{\prime}$ is integrable on $[\lambda, \mu]$ and $\alpha(t)-\alpha(s)=\int_{s}^{t} \alpha_{+}^{\prime}(r) d r$ for all $t, s \in[\lambda, \mu]$. Analogously we define an E-approximate solution $\beta=\beta(\bullet ; \lambda, \mu, w, \mathcal{E})$ to (2) on $[\lambda, \mu]$. Now let $K$ be a closed, convex (nonempty) subset of $E$. We write that $A \sim_{K} B$ if whenever $z, w \in E$ with $z-w \in K$ and $\lambda \in[0, T]$, it follows that

$$
\lim _{h \rightarrow 0+} \inf d(z-w+h(A(\lambda, z)-B(\lambda, w)) ; K) / h=0,
$$

where $d(y ; K)=\inf \{|y-x|: x \in K\}$ for each $y \in E$.

For our first result we use techniques similar to those of Martin [3] to relate the concept of $A \sim_{K} B$ to the existence of certain types of E-approximate solutions to (1) and (2).

Received by the editors July 18,1974 .

AMS (MOS) subject classifications (1970). Primary 34A40, $34 \mathrm{G} 05$.

Key words and phrases. Differential inequalities, Banach space, cones.

${ }^{1}$ This work was done while the author held a NATO Fellowship in Science. 
Proposition 1. The following statements are equivalent.

(i) $A \sim_{K} B$.

(ii) If $z, w \in E$ with $z-w \in K, \lambda \in[0, T]$ and $\varepsilon>0$, there is a $\mu=$ $\mu(z, w, \lambda, \xi) \in(\lambda, T]$ and E-approximate solutions $\alpha=\alpha(\cdot ; \lambda, \mu, z, \xi)$ to (1) and $\beta=\beta(\circ ; \lambda, \mu, w, \xi)$ to (2) such that $\alpha(t)-\beta(t) \in K$ for all $t \in[\lambda, \mu]$.

(iii) If $z, w \in E$ with $z-w \in K$ and $\lambda \in[0, T)$, then

$$
\lim _{h \rightarrow 0^{+}} d(z-w+h(A(\lambda, z)-B(\lambda, w)) ; K) / h=0 .
$$

Proof. Suppose first that (i) holds and that $z, w \in E$ with $z-w \in K$, $\lambda \in[0, T)$ and $\tilde{E}>0$. Since $A \sim_{K} B$ there is a $\delta \in(0, T-\lambda]$ and a $p \in K$ such that

$$
|z+\delta A(\lambda, z)-(w+\delta B(\lambda, w))-p| \leq \delta \mathcal{E} / 2
$$

Define $\mu=\lambda+\delta$ and define $\beta(t)=w+(t-\lambda) B(\lambda, w)$ and

$$
a(t)=\delta^{-1}[(\mu-t) z+(t-\lambda)(\beta(\mu)+p)] \quad \text { for all } t \in[\lambda, \mu] \text {. }
$$

Since

$$
\alpha(t)-\beta(t)=\delta^{-1}(\mu-t)(z-w)+\delta^{-1}(t-\lambda) p,
$$

we see that $a(t)-\beta(t) \in K$ for all $t \in[\lambda, \mu]$. Noting that $\beta(0)=w, \mid \beta(t)-$ $w|\leq \delta| B(\lambda, w) \mid$ and $\left|\beta_{+}^{\prime}(t)-B(t, \beta(t))\right|=|B(\lambda, w)-B(t, \beta(t))|$, one sees that $\delta$ can also be chosen so that $\beta$ is an $E$-approximate solution to (2) on $[\lambda, \mu]$. Also $\alpha(0)=z$ and

$$
\begin{aligned}
|\alpha(t)-z| & =\delta^{-1}(t-\lambda)|-z+\beta(\mu)+p| \\
& \leq|z-w-\delta B(\lambda, w)-p| \leq \delta \mathscr{\sigma} / 2+\delta|A(\lambda, z)|,
\end{aligned}
$$

so it may be assumed that $|A(t, a(t))-A(\lambda, z)| \leq \mathcal{E} / 2$ for all $t \in[\lambda, \mu]$ as well. Consequently,

$$
\begin{aligned}
\left|\alpha_{+}^{\prime}(t)-A(t, a(t))\right| & \leq \delta^{-1}|-z+w+\delta B(\lambda, w)+p-\delta A(\lambda, z)|+\mathcal{E} / 2 \\
& \leq \delta^{-1} \delta \mathcal{E} / 2+\widetilde{G} / 2=\mathcal{G},
\end{aligned}
$$

and so $\alpha$ is an E-approximate solution to (1) on $[\lambda, \mu]$. This shows that (i) implies (ii). Now suppose that (ii) holds and $z, w \in E$ with $z-w \in K, \lambda \epsilon$ $(0, T)$, and $\mathscr{G}>0$. If $\mu \in(\lambda, T]$ and $\alpha=\alpha(\cdot ; \lambda, \mu, z, \mathcal{E})$ and $\beta=\beta(\cdot ; \lambda, \mu, w, \mathcal{E})$ are as in (ii),

$$
\begin{aligned}
\limsup _{h \rightarrow 0+} & d(z-w+h(A(\lambda, z)-B(\lambda, w)) ; K) / h \\
\leq & \limsup _{h \rightarrow 0+}|z-w+h(A(\lambda, z)-B(\lambda, w))-(\alpha(\lambda+h)-\beta(\lambda+h))| / h \\
\leq & \limsup _{h \rightarrow 0+}|A(\lambda, z)-[\alpha(\lambda+h)-z] / h|+|B(\lambda, w)-[\beta(\lambda+h)-w] / h| \\
& =\left|A(\lambda, a(\lambda))-a_{+}^{\prime}(\lambda)\right|+\left|B(\lambda, \beta(\lambda))-\beta_{+}^{\prime}(\lambda)\right| \leq 2 \xi_{.} .
\end{aligned}
$$


Thus (ii) implies (iii). Since the assertion (iii) implies (i) is trivial, the proof is complete.

Now, for convenience, we introduce notations and suppositions which are frequently assumed to hold:

(C1) $K$ is a closed, convex subset of $E$ and $z, w \in E$ with $z-w \in K$.

(C2) $A \sim_{K} B$.

(C3) There are numbers $M>1$ and $R>0$ such that $T \leq R / M,|A(t, x)|$ $\leq M-1$ if $t \in[0, T]$ and $|x-z| \leq R$, and $|B(t, x)| \leq M-1$ if $t \in[0, T]$ and $|x-w| \leq R$.

Instead of assuming various conditions on $A$ (or $B$ ) which guarantee the existence of solutions to (1), we use the following criteria: if (C1)-(C3) hold the function $A$ is said to satisfy condition $(\mathrm{H})$ at $z$ if whenever $\left\{\mathcal{E}_{n}\right\}_{1}^{\infty}$ is a sequence in $(0, \infty)$ with $\lim _{n \rightarrow \infty} \mathcal{E}_{n}=0$ and $\alpha_{n}$ is an $\mathscr{E}_{n}$-approximate solution to (1) on $[0, T]$ with $\alpha_{n}(0)=z$, it follows that $\left\{\alpha_{n}\right\}_{1}^{\alpha}$ has a subsequence which converges uniformly to a solution $u$ to $(1)$ on $[0, T]$. An analogous definition applies to the function $B$ satisfying condition $(\mathrm{H})$ at $w$.

Remark 1. There are various conditions that one may place on $A$ in order that it satisfies condition $(\mathrm{H})$ at $z$. For example, one may assume that $A=$ $A_{1}+A_{2}$ where $A_{1}$ is completely continuous and there is a number $L>0$ such that $\left|A_{2}(t, x)-A_{2}(t, y)\right| \leq L|x-y|$ for all $t \in[0, T]$ and all $x, y \in E$ with $|x-z|,|y-z| \leq R$. Further examples involving dissipative and compactness conditions may be found in [4]. It is clear that condition (H) implies the existence of a solution $u$ to $(1)$ on $[0, T]$. Although the author is uncertain, it is probably the case that the existence of a solution to $(1)$ on $[0, T]$ does not imply that condition $(\mathrm{H})$ is satisfied.

Remark 2. Note that if (C3) holds and $\alpha$ is an E-approximate solution to (1) on $[0, S]$ with $S \leq T$ and $\mathcal{E} \in(0,1]$, then $\left|\alpha_{+}^{\prime}(t)\right| \leq|A(t, \alpha(t))|+\mathcal{E} \leq M$, and hence $|\alpha(t)-\alpha(s)| \leq M|t-s|$ for all $t, s \in[0, s]$. Similarly, if $\beta$ is an E-approximate solution to (2) on $[0, s]$ then $|\beta(t)-\beta(s)| \leq M|t-s|$ for all $t, s \in[0, s]$.

Theorem 1. Suppose that (C1)-(C3) are fulfilled, A satisfies condition $(\mathrm{H})$ at $z$, and that $v:[0, T] \rightarrow E$ is a solution to $(2)$ on $[0, T]$ with $v(0)=w$. Then there is a solution $u$ to $(1)$ on $[0, T]$ such that $u(0)=z$ and $u(t)-v(t) \in K$ for all $t \in[0, T]$.

Proof. Let $\mathscr{G}_{\epsilon}(0,1]$ and let $P_{\mathcal{E}}$ denote the class of all $\mathcal{E}$-approximate solutions $a$ to (1) on $[0, S]$ with $0 \leq S \leq T, a(S)-\nu(S) \in K$, and $d(\alpha(t)-v(t) ; K) \leq \mathscr{E}$. Partially order $P_{\mathcal{E}}$ by $\alpha_{1} \leq \alpha_{2}$ only in case $\alpha_{2}$ is an extension of $a_{1}$. Using Zorn's lemma (see also Remark 2), one easily sees that $F_{\mathcal{E}}$ has a maximal element $\alpha$. Suppose, for contradiction, that $\alpha$ is defined on $[0, S]$ with $S<T$. Using (iii) of Proposition 1 and the fact 
that $a(S)-v(S) \in K$, it follows that

$$
\begin{aligned}
\lim _{h \rightarrow 0+} & d(a(S)+h A(S, a(S))-v(S+h) ; K) / h \\
= & \lim _{h \rightarrow 0+} d\left(\alpha(S)-v(S)+h\left[A(S, a(S))-\int_{S}^{S+h} B(s, v(s)) d s\right] ; K\right) / h \\
\leq & \lim _{h \rightarrow 0+} d(\alpha(S)-v(S)+h[A(S, \alpha(S))-B(S, v(S))] ; K) / h \\
& +\lim _{h \rightarrow 0+} h^{-1} \int_{S}^{S_{+}}|B(s, v(s))-B(S, v(S))| d s \\
= &
\end{aligned}
$$

and hence there is a $\vec{\delta}>0$ and a $p \in K$ such that $S+\delta \leq T$ and

$$
|a(S)+\delta A(S, a(S))-v(S+\delta)-p| \leq \delta \mathcal{E} / 2
$$

Now define the function $\theta:[0, \delta] \rightarrow E$ by

$$
\theta(t)=\delta^{-1}[(\delta-t) \alpha(S)+t(v(S+\delta)+p)] .
$$

Then $\theta(0)=a(S)$ and $\theta(\delta)-v(S+\delta)=p \in K$. Assuming also that $\delta$ is sufficiently small so that $|\theta(t)-\alpha(S)| \leq \mathcal{E} / 2$ and $|v(S+t)-v(S)| \leq \mathcal{E} / 2$ for all $t \in[0, \delta]$, we have that

$d(\theta(t)-v(S+t) ; K) \leq|\theta(t)-\alpha(S)|+|v(S)-v(S+t)|+d(\alpha(S)-v(S) ; K) \leq \varepsilon_{.}$

Moreover, if $t \in[0, \delta]$ then

$$
\left|A(S, a(S))-\theta_{+}^{\prime}(t)\right|=\delta^{-1}|\alpha(S)+\delta A(S, a(S))-v(S+\delta)-p| \leq \mathcal{E} / 2 .
$$

In particular, $\left|\theta_{+}^{\prime}(t)\right| \leq M$ and hence $|\theta(t)-\alpha(S)| \leq M t \leq M \delta$; so we may also assume that $|A(S, a(S))-A(S+t, \theta(t))| \leq \mathcal{E} / 2$ for all $t \in[0, \delta]$. Thus $\left|\theta^{\prime}+(t)-A(S+t, \theta(t))\right| \leq \mathcal{E}$ and it follows that if $\alpha_{0}(t)=a(t)$ for $t \in[0, s]$ and $\alpha_{0}(t)=\theta(t-S)$ for $t \in[S, S+\delta]$, then $\alpha_{0} \in P_{\mathcal{E}}$ and $\alpha_{0}$ is a proper extension of $\alpha$. This contradiction shows that $\alpha$ must be defined on $[0, T]$. In particular, for each $\mathscr{E} \in(0,1]$ there is an $\mathscr{E}$-approximate solution $\alpha_{\mathcal{E}}$ to (1) on $[0, T]$ such that $d\left(\alpha_{\mathcal{G}}(t) \ldots v(t) ; K\right) \leq \mathcal{E}$. Using the fact that $A$ satisfies condition $(\mathrm{H})$ at $z$ it now follows immediately that there is a solution $u$ to (1) on $[0, T]$ such that $u(t)-v(t) \in K$ for all $t \in[0, T]$, and the proof is complete.

Remark 3. Note, that with the suppositions of Theorem 1, one can be assured only that there is at least one solution $u$ to (1) on $[0, T]$ such that $u(t)-v(t) \in K$ for all $t \in[0, T]$. As can be shown by very simple examples, it is not necessarily the case that every solution of (1) must satisfy this relationship with $v$.

As a simple example to illustrate Theorem 1 , let $\eta$ be a positive number and take $K=\{x \in E:|x| \leq \eta\}$. Let $F$ be the duality mapping from $E$ into the class of subsets of the dual space $E^{*}$ of $E$ (i.e., $F x=\left\{f \in E^{*}: f(x)=\right.$ 
$\left.|x|^{2}=|f|^{2}\right\}$ for each $\left.x \in E\right)$. Then $A \sim_{K} B$ only in case $\operatorname{Re} f(A(\lambda, x)-B(\lambda, y))$ $\leq 0$ however $\lambda \in[0, T], x, y \in E$ with $|x-y|=\eta$, and $f \in F(x-y)$.

Of course, the most important examples of convex sets $K$ are provided by cones. We say that $K$ is a closed cone in $E$ if $K$ is closed and whenever $x, y \in K$ and $a \geq 0$, it follows that $x+y \in K$ and $a x \in K$. The above results can be improved somewhat when $K$ is a cone; so, in place of (C1), we assume the following condition:

$(\mathrm{C} 1)^{\prime} K$ is a closed cone in $E$ and $z, w \in E$ with $z-w \in K$. Moreover, for notational convenience, if $x, y \in E$ with $x-y \in K$, we write $x \geq$ $y$ or $y \leq x$. In this case we have the following analogous result to Theorem 1:

Theorem 2. Suppose that (C1)', (C2) and (C3) are fulfilled, A satisfies condition $(\mathrm{H})$ at $z$, and that $v:[0, T] \rightarrow E$ is continuously differentiable with $v(0)=w$ and $v^{\prime}(t) \leq B(t, v(t))$ for all $t \in[0, T]$. Then there is a solution $u$ to (1) on $[0, T]$ such that $u(0)=z$ and $u(t) \geq v(t)$ for all $t \in[0, T]$.

Proofo Set $g(t)=B(t, v(t))-v^{\prime}(t)$ for all $t \in[0, t]$ and define

$$
B_{1}(t, x)=B(t, x)-g(t) \text { for all }(t, x) \in[0, T] \times E \text {. }
$$

One has that $v^{\prime}(t)=B_{1}(t, v(t))$ for all $t \in[0, T]$ and hence Theorem 2 will be a direct consequence of Theorem 1 once it is shown that $A \sim_{K} B_{1}$. Note that, since $K$ is a cone, if $x \in E$ and $y \in K$ then $d(x+y ; K) \leq d(x ; K)$ (for if $\left\{p_{n}\right\}_{1}^{\infty}$ is a sequence in $K$ such that $d(x ; K)=\lim _{n \rightarrow \infty}\left|x-p_{n}\right|$, then $p_{n}+y \in K$ and hence $\left.d(x+y ; K) \leq \lim _{n \rightarrow \infty}\left|(x+y)-\left(p_{n}+y\right)\right|=d(x ; K)\right)$. Since $h g(t) \in K$ for all $b>0$ and $t \in[0, T]$, we see that if $x \geq y$ and $t \in[0, T]$,

$$
\begin{aligned}
\lim _{h \rightarrow 0^{+}} & d\left(x-y+h\left[A(t, x)-B_{1}(t, y)\right] ; K\right) / h \\
& =\lim _{h \rightarrow 0+} d(x-y+h[A(t, x)-B(t, y)]+h g(t) ; K) / h \\
& \leq \lim _{h \rightarrow 0^{+}} d(x-y+h[A(t, x)-B(t, y)] ; K) / h=0 .
\end{aligned}
$$

Thus $A \sim_{K} B_{1}$ and the proof of Theorem 2 is complete.

Remark 4. Theorem 2 is not true for general convex subsets $K$ of $E$. As a simple example, take $K=\{x \in E:|x| \leq 1\}$ and define $A(t, x)=B(t, x)$ $=\theta$ for all $(t, x) \in[0,1] \times E$. Set $z=0$ and let $w \in E$ be such that $|w|=1$. Define $v(t)=(1+t) w$ for all $t \in[0,1]$. Then $B(t, v(t))-v^{\prime}(t)=-w \in K$ and, since $u(t)=\theta$ for all $t \in[0,1]$, we see that $u(t)-v(t)=-(1+t) w \notin K$ and $v(t)-u(t)=(1+t) w \notin K$ for any $t \in(0,1]$.

In the case that one imposes further smoothness conditions on $A$ and $B$, an additional result may be obtained. In the theorem below, we let $F$ denote the duality mapping from $E$ into the class of subsets of $E^{*}$ (see the paragraph following Remark 3).

Theorem 3. Suppose that $(\mathrm{C} 1)^{\prime}$ holds, $A \sim_{K} A, A \sim_{K} B$ and $B \sim_{K} B$. 
Suppose further that there is a number $L>0$ such that

(4) $\operatorname{Re} f[A(t, x)-A(t, y)] \leq L|x-y|^{2}$ and $\operatorname{Re} f[B(t, x)-B(t, y)] \leq L|x-y|^{2}$ for all $t \in[0, T], x, y \in E$, and $f \in F(x-y)$. Also, let $u, v:[0, T] \rightarrow E$ be continuously differentiable with $u(0)=z, v(0)=w$ and

$$
u^{\prime}(t)-A(t, u(t)) \geq v^{\prime}(t)-B(t, v(t)) \text { for all } t \in[0, T] .
$$

Then $u(t) \geq v(t)$ for all $t \in[0, T]$.

Indication of proof. By using a standard argument, it is enough to show that if $0 \leq \lambda<T$ and $u(t) \geq v(t)$ for all $t \in[0, \lambda]$, then there is a $\delta>0$ such that $\lambda+\delta \leq T$ and $u(t) \geq v(t)$ for all $t \in[\lambda, \lambda+\delta]$. So assume that $u(t) \geq v(t)$ for all $t \in[0, \lambda]$ and that $0 \leq \lambda<T$. Define $g_{1}(t)=v^{\prime}(t)-B(t, v(t))$ for all $t \in[\lambda, T]$ and $A_{1}(t, x)=A(t, x)+g_{1}(t)$ for all $(t, x) \in[\lambda, T] \times E$. Set $K_{-}=$ $\{-x: x \in K\}$ and note that if $t \in[\lambda, T)$ and $x, y \in E$ with $x-y \in K_{-}$, then $y-x \in K$ and

$$
\begin{aligned}
\lim _{h \rightarrow 0+} & d\left(x-y+h\left[A_{1}(t, x)-A_{1}(t, y)\right] ; K_{-}\right) / h \\
& =\lim _{h \rightarrow 0^{+}} d(y-x+h[A(t, y)-A(t, x)] ; K) / h=0 .
\end{aligned}
$$

Thus $A_{1} \sim_{K_{-}} A_{1}$. The dissipative condition (4) on $A$, and hence on $A_{1}$, shows that $A_{1}$ satisfies condition $(\mathrm{H})$ on $[\lambda, \lambda+\delta]$ at $u(\lambda)$ for some $\delta>$ 0 , and also that the solution to the initial value problem $u_{1}^{\prime}(t)=A_{1}(t, u(t))$, $u_{1}(\lambda)=u(\lambda), t \in[\lambda, \lambda+\delta]$ is unique. Thus, by Theorem 2 with $K$ replaced by $K_{-}, A=B=A_{1}$, and $z=w=u(\lambda)$, we see that $u_{1}(t)-u(t) \in K$ and hence

$$
u(t) \geq u_{1}(t) \text { for all } t \in[\lambda, \lambda+\delta]
$$

where $u_{1}^{\prime}(t)=A_{1}\left(t, u_{1}(t)\right), u_{1}(\lambda)=u(\lambda)$ and $t \in[\lambda, \lambda+\delta]$. Similarly, defining $g_{2}(t)=u^{\prime}(t)-A(t, u(t))$ and $B_{1}(t, x)=B(t, x)+g_{2}(t)$ for all $t \in[\lambda, \lambda+\delta]$ and all $x \in E$, we see that $B_{1} \sim_{K} B_{1}$ and $v^{\prime}(t) \leq B_{1}(t, v(t))$ for all $t \epsilon$ $[\lambda, \lambda+\delta]$. Again using (4) and applying Theorem 2 with $A=B=B_{1}$ and $z=w=v(\lambda)$, we see that

$$
v(t) \leq v_{1}(t) \text { for all } t \in[\lambda, \lambda+\delta]
$$

where $v_{1}^{o}(t)=B_{1}\left(t, v_{1}(t)\right), v_{1}(\lambda)=v(\lambda)$ and $t \in[\lambda, \lambda+\delta]$. Now let $x, y \in E$ with $x \geq y$. Noting that $g_{1}(t) \geq g_{2}(t)$ we have that $g_{1}(t)-g_{2}(t) \in K$ and hence, since $A \sim_{K} B$,

$$
\begin{aligned}
\lim _{h \rightarrow 0+} & d\left(x-y+h\left[A_{1}(t, x)-B_{1}(t, x)\right] ; K\right) / h \\
& =\lim _{h \rightarrow 0^{+}} d\left(x-y+h[A(t, x)-B(t, x)]+h\left[g_{1}(t)-g_{2}(t)\right] ; K\right) / h \\
& \leq \lim _{h \rightarrow 0^{+}} d(x-y+h[A(t, x)-B(t, x)] ; K) / h \\
& =0 .
\end{aligned}
$$


Thus $A_{1} \sim_{K} B_{1}$ and Theorem 1 with $A=A_{1}$ and $B=B_{1}$ implies since solutions are unique that

$$
u_{1}(t) \geq v_{1}(t) \text { for all } t \in[\lambda, \lambda+\delta]
$$

Inequalities (5), (6) and (7) show that $u(t) \geq v(t)$ for all $t \in[\lambda, \lambda+\delta]$ and the assertion of Theorem 3 now follows.

Remark 5. When $A=B$ in Theorem 3 and the dissipative assumption (4) is replaced by a Lipschitz condition, then we obtain a result of Volkmann [7]. The proof techniques here and those of Volkmann [7] are considerably different, however.

Remark 6. The definition of $A \sim_{K} B$ (see equation (3)) can also be expressed in terms of inequalities involving members of the dual space $E^{*}$ of $E$. If $K$ is a closed convex subset of $E$ and $z, w \in E$ with $z-w \in \partial K$ (where $\partial K$ is the boundary of $K)$, then (3) holds only in case $\operatorname{Re} f(A(\lambda, z)-B(\lambda, w)) \leq 0$ whenever $f \in E^{*}$ with the property that $f(z-w)=r \geq 0$ and $\operatorname{Re} f(x) \leq r$ for all $x \in K$. See Redheffer and Walter [5]. See also Köthe [2, p. 345].

Remark 7. The results of Proposition 1 and Theorem 1 are valid if one assumes only that $K$ is a closed subset of $E$. However, one needs an alteration in (ii) of Proposition 1. Instead of assuming that $\alpha(t)-\beta(t) \in K$ for all $t \in[\lambda, \mu]$, we assume that $\alpha(\mu)-\beta(\mu) \in K$ and for each $t \in[\lambda, \mu)$ there is an $\eta(t) \in[\lambda, t]$ such that $t-\eta(t) \leq \mathcal{E}$ and $\alpha(\eta(t))-\beta(\eta(t)) \in K$.

Acknowledgment. The author is most appreciative to Dr. Peter Volkmann for several helpful conversations during the Conference on Differential Equations held in Dundee, Scotland, from March 26-29, 1974.

\section{REFERENCES}

1. L. Bitner, Die elementaren Differentialo und Integralungleichungen mit einem allgemeinen Ungleichungsbegriff, Math. Nachr. 38 (1968), 1-17. MR 39 \#589.

2. G. Köthe, Topologische linear Räume. I, 2nd ed., Die Grundlehren der math. Wissenschaften, Band 107, Springer-Verlag, Berlin, 1966; English transl., Die Grundlehren der math. Wissenschaften, Band 159, Springer-Verlag, New York, 1969. MR 33 \#3069; $40 \# 1750$.

3. R. Martin, Approximation and existence of solutions to ordinary differential equations in Banach spaces, Funkcial. Ekvac. 16 (1973), 195-213.

4. - Remarks on ordinary differential equations involving dissipative and compact operators, J. London Math. Soc. 10 (1975), 61-65.

5. R. Redheffer and W. Walter, Flow-invariant sets and differential inequalities in normed spaces, Applicable Anal. (to appear).

6. P. Volkmann, Ger!ühnliche Differentialungleichungen mit quasimonoton wachsenden Funktionen in topologischen Vektorräumen, Math. Z. 127 (1972), 157164. MR $46 \# 7661$.

7. _- Gewöhnliche Differentialungleichungen mit quasimonoton wachsenden Funktionen in Banachräumen, Proc. Conf. on Differential Equations (Dundee, Scotland, 1974) (to appear).

DEPARTMENT OF MATHEMATICS, NORTH CAROLINA STATE UNIVERISTY, RALEIGH, NORTH CAROLINA 27607

MATHEMATICS DIVISION, UNIVERSITY OF SUSSEX, FALMER, BRIGHTON BN1 9QH, ENGLAND 